\title{
Study of Clinico Radiological Profile of Ischemic Cerebrovascular Stroke and Its Outcome at Tertiary Care Centre
}

\author{
Vaishali Patel ${ }^{1}$, Asha Vagadiya² \\ ${ }^{1}$ Associate Professor of Medicine, Medical College, Vadodara, ${ }^{2}$ Tutor of Physiology, Medical College, Vadodara.
}

\section{Abstract}

Background: Ischemic CV stroke is disease of long term Disability and Death accounts for $>80 \%$ of total stroke events. The main objective of the study was to study clinical and radiological profile, risk factors and outcome of stroke. Subjects and Methods: An observational study of 46 patients of acute ischemic cerebrovascular stroke was conducted during January 2014 to November 2014. All patients were subjected to Sociodemographic, Clinical, family h/o risk factors, dietary, other lifestyle habits and examination with written consent. Apart from routine blood investigations, special investigations like Uric acid, urinary albumin creatinine ratio, ECG,2D Echo, Carotid Doppler, CT Scan/MRI brain were done within24 hours of onset. NIHSS and MRS scores were used for severity assessment. Results: Majority of patients were in 5564 years $(30.4 \%)$ age group with male predominance $(52 \%)$ (mean age 55.52 \pm 12.61 ). Old aged patients are more likely to be selected for study. Microalbuminuria was found on $34(73.9 \%)$ Patients with recent stroke. $52.1 \%$ patients having serum uric acid $>8.36 .9 \%$ patients. ECG suggested LVH and normal findings in $36.9 \%$ while $15.2 \%$ and $10.8 \%$ had stroke related changes and myocardial ischemia. Majority 30 $(65 \%)$ of patients showed concentric hypertrophy, followed by diastolic dysfunction $11(23 \%)$ on $2 \mathrm{D}$ Echo. Atherosclerotic changes seen in $60.8 \%$ on carotid Doppler study. majority $32.6 \%$ patients were affected in MCA territory, followed by $23.9 \%$ PCA territory followed by $19.5 \%$ infarct in thalamus. NIHSS and MRSmean score was $20.2 \pm 12$ and 3.9+1.8 on admission and $15.7 \pm 13$ and $3.23+1.91$ on discharge respectively. Hospital outcome was good. No mortality during entire study period. Conclusion: Stroke a high socioeconomic burden in community. Apart from Age and hypertension, microalbuminuria and uric acid may be considered as independent risk factors for ischemic stroke. Identification and treatment of risk factors can prevent stroke related morbidity and mortality.

Keywords: Ischemic cerebrovascular stroke, Atherosclerotic risk factors.

Corresponding Author: Dr. Asha B. Vagadiya, Tutor of Physiology, Medical College, Vadodara.

Received: December 2019

Accepted: December 2019

\section{Introduction}

Stroke is defined as rapidly developing clinical signs of focal or global disturbance of cerebral function lasting for more than 24 hours with no obvious cause other than vascular origin. $^{[1]}$ Astroke or cerebrovascular accident idefined by abrupt onset of a neurological deficit that is attributable to a focal vascular cause. Thus the definition of stroke is clinical and laboratory studies including brain imaging are used to support the diagnosis. Cerebral ischemia is caused by reduction in blood flow lasting for more than several seconds. If cessation of flow lasts for more than few minutes, infarction or death of brain tissue results. ${ }^{[2]}$ In India,community survey has shown a crude prevalence rate of hemiplegia 200 per 1 lac persons, nearly $1.5 \%$ of all urban hospital admissions, $4.5 \%$ of all medical and $20 \%$ of neurological cases ${ }^{[3]}$ Stroke incidence rises steeply with age; stroke in young is less commom as compared to older but is of great concern due associated mortality and morbidity. ${ }^{[4]}$ Stroke is second most common cause of disability and dementia in adults > 65 years worldwide; close to $25 \%$ stroke survivors develop dementia.[5] Thus stroke is an important cause of morbidity and long term disability.

\section{Subjects and Methods}

The study was conducted in department of medicine, Medical College and SSG Hospital, Vadodara during January 2014 to November 2014 after approval of medicine department and Institutional ethics committe. The study comprises of 46 patients with history and clinical features suggestive of acute ischemic cerebrovascular stroke admitted in Medicine wards applying inclusion and exclusion criteria. A Written Informed consent obtained from all patients.

\section{Inclusion Criteria}

a. Age> 25 yrs

b. First ever in time acute ischemic stroke within 72 hours of onset of symptoms

c. CT Scan evidence of Brain infarction within $24 \mathrm{hrs}$ of onset of symptoms.

\section{Exclusion Criteria}
a. Past with h/o TIA/ Stroke
b. CT scan evidence of haemorrhage or other space occupying lesions other than infarction
c. known case of cardiac Diseases, Hypertension, 
Patel d Vagadiya : Clinica Radialagical Prapile of Jschemic Cerebravascular Strake

Diabetes Mellitus, Nephropahy

d. haematological abnormalities like leukemia, polycythemia or other myeloproliferative diseases

e. Neoplastic Disease

f. Connective tissue disorder

g. Systemic infections including Bacterial meningitis.

h. Prothrombotic states, Sickle cell Disease, Protein C/S deficiency,

Detailed clinical history obtained about symptoms. They were specifically asked for symptoms of headache, vomiting,vertigo, limb weakness, gait imbalance, speech disturbance,sensory symptoms, visual complaints among the other symptoms. Past and family history especially for risk factors(i.e hypertension, diabetes mellitus, ischemic heart disease and TIA/Stroke) and treatment for the same. All patients were evaluated for clinical examination mainly CNS. The severity of stroke assessed using NIHSS (National Institute of Health Stroke score, De Graba, Hallenbeck. NIHSS and outcome 1999 ) for all the patients at admission with total score range from 0-42 with higher values representing more severe infarcts. The MRS (Modified Rankin Scale) was used to assess the outcome of patients (0-6). With higher the score, worse is the prognosis .All the pateints blood samples were taken within $24 \mathrm{hrs}$ of stroke with written consent and sent for biochemical analysis. Sampleswere analysed in laboratory of Biochemistry Department using standard analyzer. All blood samples were sent for complete hemogram. Urine analysis, Random blood sugar, Renal and liver function tests, ECG, fundus examination, Carotid Colour Doppler, CT/ MRI scan examination,lipid profile, 2D Echo, USG KUB. Spot urine samples to measure the urinary albumin to creatinine ratio(ACR)obtained of all patients. A ratio of $<0.3$ is considered as normal while the value between 0.3 to 3.0 is considered as microalbuminuria. The patients with IFG or IGT were not included as diabetics in this study. All data were analysedusing Microsoft excel software .Statistical analysis of data was performed in form of mean and standard deviation.t- test was applied to test statistically significant difference in groups. Two tailed $\mathrm{P}$ value $<0.05$ were considered significant.

Results

Table 1: Age distribution among the study population

\begin{tabular}{|l|l|l|}
\hline Age distribution (years) & Frequency & Percentage \\
\hline 25 to 34 & 2 & 4.3 \\
\hline 35 to 44 & 5 & 10.8 \\
\hline 45 to 54 & 11 & 23.9 \\
\hline 55 to 64 & 14 & 30.4 \\
\hline 65 to 74 & 11 & 23.9 \\
\hline 75 to 84 & 2 & 4.3 \\
\hline$>85$ & 1 & 2.2 \\
\hline TOTAL & 46 & 100 \\
\hline
\end{tabular}

In our study,out of 46 patients,maximum numbers of patients were in 55-64 years (30.4\%) of age with male predominance $(52 \%)$ was observed.
Table 2: Distribution according to motor weakness

\begin{tabular}{|l|l|l|}
\hline Limbs Involved & Frequency & Percentage \\
\hline Hemiplegia & 34 & $74 \%$ \\
\hline Bilateral & 5 & $10.9 \%$ \\
\hline Monoplegia & 1 & $2.1 \%$ \\
\hline No Motor weakness & 6 & $13 \%$ \\
\hline Total & 46 & $100 \%$ \\
\hline
\end{tabular}

In our study, overall $34(74 \%)$ patients presented with hemiplegia.56.5\% patients presented with right hemiplegia , $17.5 \%$ with left hemiplegia, $10.9 \%$ with bilateral motor weakness while $13 \%$ presented with no motor weakness.

Table 3: Distribution according to Microalbuminuria

\begin{tabular}{|l|l|l|}
\hline Microalbuminuria & Frequency & Percentage \\
\hline Present & 34 & $73.91 \%$ \\
\hline Absent & 12 & $26.09 \%$ \\
\hline Total & 46 & $100 \%$ \\
\hline
\end{tabular}

[Table 3] Shows microalbuminuria was found on 34 (73.9\%) patient with recent ischemic stroke while $12(26 \%)$ had no microalbuminuria.

Table 4: Distribution according to Serum Uric Acid level

\begin{tabular}{|l|l|l|}
\hline Serum Uric acid & Frequency & Percentage \\
\hline$<2$ & 10 & 21.7 \\
\hline 2 to 8 & 12 & 26 \\
\hline$>8$ & 24 & 52.1 \\
\hline Total & 46 & 100 \\
\hline
\end{tabular}

[Table 4] shows that $52.1 \%$ patients having serum uric acid $>8$ while $26 \%$ and $21.7 \%$ were having serum uric acid $<2$ and 2 to 8 respectively.

Table 5: Distribution according to ECG changes

\begin{tabular}{|l|l|l|}
\hline ECG & Frequency & Percentage \\
\hline Ischemic & 5 & 10.8 \\
\hline LVH & 17 & 36.9 \\
\hline Stroke related changes & 7 & 15.2 \\
\hline Normal & 17 & 36.9 \\
\hline Total & 46 & 100 \\
\hline
\end{tabular}

In present study, overall $36.9 \%$ patients ECG suggested LVH and normal finding while $15.2 \%$ and $10.8 \%$ had stroke related changes and myocardial ischemia.

Table 6: Distribution according to 2D ECHO

\begin{tabular}{|l|l|l|}
\hline Findings & Frequency & Percentage \\
\hline Concentric hypertrophy & 30 & $65 \%$ \\
\hline Diastolic dysfunction & 11 & $23 \%$ \\
\hline Normal & 5 & $12 \%$ \\
\hline Total & 46 & 100 \\
\hline
\end{tabular}

Table shows the 2D Echo finding, where it was seen that majority 30(65\%) of patients showed concentric hypertrophy , followed by diastolic dysfunction $11(23 \%)$ and $5(12 \%)$ patients showed normal echocardiogram.

Table 7: Distribution according to Carotid Colour Doppler findings

Carotid Colour Doppler

Atherosclerotic changes

Normal

\begin{tabular}{|l|l|}
\hline Frequency & Percentage \\
\hline 28 & 60.8 \\
\hline 18 & 39.2 \\
\hline
\end{tabular}

In present study, carotid Doppler revealed atherosclerotic changes seen in $60.8 \%$ of ischemic cerebrovascular stroke. 
Table 8: Distribution according to radiological finding on

Neuro imaging(CT Scan/MRI)

\begin{tabular}{|l|l|l|}
\hline CT Scan & No. of patients & Percentage \\
\hline MCA territory & 15 & 32.6 \\
\hline PCA territory & 11 & 23.9 \\
\hline Infarct in thalamus & 9 & 19.5 \\
\hline $\begin{array}{l}\text { Infarct in internal } \\
\text { capsule }\end{array}$ & 6 & 13 \\
\hline $\begin{array}{l}\text { Infarct in caudate } \\
\text { nucleus }\end{array}$ & 4 & 8.6 \\
\hline Infarct in cerebellum & 1 & 2.2 \\
\hline Total & 46 & $100 \%$ \\
\hline
\end{tabular}

In our study, majority $32.6 \%$ patients were affected in MCA territory, followed by $23.9 \%$ PCA territory followed by 19.5 $\%$ infarct in thalamus, followed by $13 \%$ in internal capsule while $8.6 \%$ and $2.2 \%$ seen in both caudate nucleus and cerebellum

Table 9: Distribution according to NIHSS Score

\begin{tabular}{|l|l|l|}
\hline NIHSS & Frequency & Percentage \\
\hline$<10$ & 27 & 58.7 \\
\hline$\geq 10$ & 19 & 41.3 \\
\hline Total & 46 & 100 \\
\hline
\end{tabular}

In our study, 27(58.7\%) had NIHSS Score $<10$ while $19(41.3 \%)$ had score $>10$.

Furthermore, 17 out of 19 patients with NIHSS Score more than or equal to 10 had microalbuminuria while only 2 patients did not have microalbuminuria. NIHSS mean score was $20.2 \pm 12$ on admission and $15.7 \pm 13$ on discharge with $\mathrm{p}$ value less than 0.05 significant.

Furthermore, the presence of microalbuminuria with higher score represent poor prognosis as compared to absent microalbuminuria.

Table 10: Distribution according to MRS Score

\begin{tabular}{|l|l|l|}
\hline MRS & Frequency & Percentage \\
\hline$\leq 3$ & 26 & 56.6 \\
\hline$>3$ & 20 & 43.4 \\
\hline Total & 46 & 100 \\
\hline
\end{tabular}

In our study, 20 patients had MRS Score more than 3. Furthermore 18 out of 20 had microalbuminuria, only 2 patients did not have microalbuminuria.

\section{Discussion}

Age is the most common non modifiable risk factor for the development of stroke. In current study most of the patients $30.4 \%$ were belonging to $55-64$ years followed by $23.9 \%$ in 65-74 and 45-54 years age group equally. Among the patients, youngest patient was 27 years old and oldest patient was 85 years old. The mean age was $55.52 \pm 12.61$ years .our study correlates well with Rathman et $\mathrm{al}^{[6]}$ study and vivekjain et al study. ${ }^{[7]}$

In present study, motor weakness in the form of hemiplegia with upper motor neuron facial paresis was the most common symptom. Right hemiplegia was the commoner one, seen in $56.5 \%$ followed by left hemiplegia. Putaala et $\mathrm{al}^{[8]}$ studied 1008 ischemic stroke patients reported that incidence of right hemiplegia was $60 \%$.

In our study.73.91\% had microalbuminuria suggest new onset stroke has more likely to have microalbuminuria.the finding is consistent with Turaj et $\mathrm{al}^{[9]}$ and Slowik et $\mathrm{al}^{[10]}$ studies.
In present study,52.1\% patients having serum uric acid $>8$ $\mathrm{mg} / \mathrm{dl}$.In Millinois et $\mathrm{al}^{[11,12]}$ study,concluded that $\mathrm{s}$ erum uric acid is associated with increased risk for acute ischemic stroke in elder patients independent of concurrent metabolic derangements. In Strorhaug et $\mathrm{al}^{[13]}$ study, a large study has demonstrated an independent association of serum uric acid with cerebrovascular storke in patients age 45 years regardless of sex, presence of CV Disease or race. Our findings correlate well with above studies.

In our study,36.9 had ECG changes of LVH suggesting Hypertension while $15.2 \%$ had myocardial ischemia. $\mathrm{T}$ in present study it is seen that incidence of LVH, HTN and IHD more frequent in males as compared to females.all patients were males except two females who had diagnosed to have hyperhomocystenemia after exclusion of all other causes. Thus hypertension is most important cause for cerebrovascular stroke. Our finding consistent with Mykkanen et $\mathrm{al}^{[14]}$ and Kumar $\mathrm{H}$ etal ${ }^{[15]}$ studies.

In 2D Echo finding, majority $30(65 \%)$ of patients showed concentric hypertrophy, followed by diastolic dysfunction $11(23 \%)$ which also favours evidence of long standing hypertension.

Doppler atherosclerosis changes seen in 56\% in Dinesh et $\mathrm{al}^{[16]}$ study of 177 cases.

In CT Scan, within six hours of the onset of ischemic stroke,most patients will have a normal Computed tomography scan while After 6-12 hours, hypodensity area seen. Cerebral infarction results from the atherosclerotic obstruction of large cervical and cerebral arteries with ischemia in all or part of the territory of the occluded artery. Lacunar cerebral infarction is small deep infarcts in the territory of small penetrating arteries due to a local disease of these vessels, mainly related to chronic Hypertension.

In present study carotid and ICA atherosclerosis and thrombosis was seen in 61\%.CT- atherosclerosis thrombosis were seen in $61 \%$ cases related to mostly hypertension .Extensive thrombosis intracranial as well as extracranial arteries either with common carotid or ICA were seen in 52 $\%$.Most of them related to atherosclerotic changes in major vessels, and also seen in carotid colour Doppler studies.Lacunar infarct were seen in \% cases in present study.

In our study, NIHSS mean score was $20.2 \pm 12$ on admission and $15.7 \pm 13$ on discharge with $p$ value less than 0.05 significant. 17 patients had NIHSS Score more than or equal to 10 had microalbuminuria with $p$ value less than 0.05 .Thus there is significant correlation between microalbuminuria and NIHSS Score on admission. The presence of microalbuminuria is associated with higher NIHSS Score, represents a more severe infarct and poor prognosis as well. Our study finding consistent with Gumbinger et $\mathrm{al}^{[17]}$ study. In our study, 20 had MRS Score more than 3 . The MRS Score was $3.9 \pm 1.8$ while on discharge $3.23 \pm 1.91$ with definite improvement with $\mathrm{p}$ value <o.o1 which is statistically significant. Furthermore, 18out of 20 had microalbuminuria. The presence of microalbuminuria is associated with higher MRS Score representing poor prognosis. $\mathrm{P}$ value was less than 0.05 indicating significant correlation between microalbuminuria and high MRS Score,thus suggest poor outcome.Our finding consistent with Cho et al ${ }^{[18]}$ study.

The ability to predict clinical improvement and deficit 
progression in patients with acute ischemic infarct can be a valuable asset in future attempt to assess the therapeutic intervention. It can provide a guide to expected outcome when considering subject inclusion and sample size in therapeutic trials. Additionally it can provide insight into potential ongoing injury that occur after the initial ischemic insult.

\section{Conclusion}

Our study confirms that age and hypertension are important atherosclerotic risk factors for ischemic cerebrovascular stroke. Hypertension being one of the major risk factor leading to stroke also supported by evidence on Electrocardiogram and 2D Echocardiography . Even serum uric acid and Microalbuminuria may be considered as leading risk factors for cerebrovascular stroke.

Our study demonstrated potential value of NIHSS and MRS score in identifying those patients who are likely to progress as well as improving compared on admission and discharge. This can provide new window to deliver therapies targeting these sequela of ischemia.

\section{Limitation of Study}

1. The study has not included those patients whose prognosis might have too poor to reach hospital on time or good enough with minimal impairment to seek medical consultation at tertiary care centre.

2. The large study sample size is ideally required for the statistical significance of the results, their implications and validity to extrapolate result and to suggest recommendation on the basis of same.

3. The study involved only short term follow up hence long term prognosis can be more justified for Prognostification

\section{Acknowledgement}

We express our sincere thanks to Medicine Department, SSG Hospital, Vadodara for permission to carry out study. I am thankful to all respected teachers who guided conducting the study. Lastly I express my gratitude to my Patients for their Participation.

\section{References}

1. Hatano S. Experience from a multicenter Stroke Register,: A Preliminary Report, Bulletin WHO 1976;54:541-4.

2. Longo, Kasper, Hauser, Jameson, Loscalzo.Harrison's Principles of Internal Medicine, 20 thediton, New York USA,:McGraw-Hill Companies;2018; vol 2, p-3068

3. Association of Physician of India(API), textbook of medicine, $9^{\text {th }}$ edition,vol2 ,page.1401.

4. Putaala J, Metso AJ, Metso TM, Konkola Y et al. Analysis of 1008 consecutive patients with first ever stroke, The Helnski young stroke registry.,Stroke 2009; vol 40(4):1195-1203.

5. Libre J, ValhuerdiA,Fernandez O. Prevalence of stroke and associated risk factors in older adults in Havana city and Matanzas Provinus, Cuba (10/66 population based study) MEDICC Review 2012;12;2024.

6. Rathmann W, Funkhouser E, Dyer AR, RosemanJM.The CARDIA (Coronary artery risk development in young adults)Study, Ann Epidemiol 1998;8;250-261

7. Vivekjain, shaikhMks,siddhantjain and mukeshmeena .comparative study of serum vitamin D levels and other biomarkers in patients attending Tertiary cardiac care center.int. j.bioassays, 2015;4(04);3812-14.

8. PutaalaJukka, Antti J,Metso, Tiina M. Metso, Nina Konkola, Yvonn Kraemer , Elena. Stroke: The Helsinki Young Stroke Registry 2010.DOI: 10.1161 / STROKEAHA.108.529883.

9. WojeiechTuraj et al. The prognostic significance of microalbuminuria in non-diabetic acute stroke patients. Med SciMonit 2001;7(5):989-994.

10. Slowik A et al. Microalbuminuria in non-diabetic acute stroke patients with acute ischemic stroke: Prevalence, clinical correlates and prognostic significance. Cerebrovascular Dis 2002;14(1):15-21.

11. Milinois, Haralampos J. et al "Serum uric acid levels and risk for acute ischemic nonembolic stroke in elderly subjects." Journal of internal medicine. 258.5 (2005):435-441.

12. Milinois, H.J et al, "Risk factors for first ever acute ischemic nonembolic stroke in elderly individuals" International journal of Cardiology 99.2 (2005):269-275

13. Storhaug, Hilde $\mathrm{M}$ et al.Uric acid is a risk factor for ischemic stroke and all cause mortality in the general population:a gender specific analysis from The Tromso Study. BMC cardiovascular disorders 2013,13.1;115

14. Mykkanen L et al.Microalbuminuria and carotid artery intima media thickness in non diabetic and NIDDM Subjects. The Insulin resistence Atherosclerosis Study (IRAS) Stroke 1997;28:1710-16.

15. Kumar G. Kalita B, Bansal V, Jain SK, Misra U. Magnetic resonance angiography findings in patients with ischemic stroke from north india.Stroke cerebrovascular Dis.2010 Mar 19(2): 146-52.

16. Dineshnayak, murlidharan, k. radhakrishnan. The national medical journal of indiavol 10,no.3,may 1997:10:107-12etal for athero changes in stroke.

17. CGumbinger, M.Sykora, J. Diedler, P. Ringleb, A. Rocco: Microalbuminuria : A potential prognostic marker for acute stroke:2012:83:1357-1360.

18. Cho BH, Kim JT, Chang J, Choi KH, Nam TS, Choi SM, Leee SH, Park MS.et al.Early clinical implications of microalbuminuria in patients with acute ischemic stroke.

Copyright: () the author(s), 2019. It is an open-access article distributed under the terms of the Creative Commons Attribution License (CC BY 4.0), which permits authors to retain ownership of the copyright for their content, and allow anyone to download, reuse, reprint, modify, distribute and/or copy the content as long as the original authors and source are cited.

How to cite this article: Patel V, Vagadiya A. Study of Clinico Radiological Profile of Ischemic Cerebrovascular Stroke and Its Outcome at Tertiary Care Centre. Acad. J Med. 2019;2(2):159-162.

DOI: dx.doi.org/10.21276/ajm.2019.2.2.41

Source of Support: Nil, Conflict of Interest: None declared. 